\title{
HIF-1 expression in retinal ganglion cells and optic nerve axons in glaucoma
}

\author{
Joanna Reszeć ${ }^{1}$, Renata Zalewska ${ }^{2}$, Piotr Bernaczyk ${ }^{1}$, Lech Chyczewski ${ }^{1}$ \\ ${ }^{1}$ Department of Medical Pathomorphology, Medical University of Bialystok, Poland \\ ${ }^{2}$ Department of Ophthalmology, Medical University of Bialystok, Poland
}

\begin{abstract}
Glaucoma is a result of increased intraocular pressure leading to damage to retinal ganglion cells and optic nerve axons. The aim of this study was to evaluate HIF-1 expression in optic nerve axons and retinal ganglion cells in 42 eyes enucleated because of complete glaucoma compared to eyes removed because of injury. The immunohistochemical reaction was done and specimens were examined under a light microscope. $57 \%$ of cases presented HIF-1 expression in the optic nerve axons, and $52.3 \%$ in the retinal ganglion cells. 20 out of 42 $(47.6 \%)$ cases were HIF-1 positive both in the optic nerve axons and in the retinal ganglion cells, and the staining was evident mostly in the nuclear and perinuclear area. Our present results indicate that HIF- 1 expression in hypoxic conditions in glaucoma might be a very crucial stage in damage to retinal ganglion cells and optic nerve axons, and might be a successful target for the implementation of neuroprotective drugs. (Folia Histochemica et Cytobiologica 2012, Vol. 50, No. 3, 456-459)
\end{abstract}

Key words: optic nerve axons, retinal ganglion cells, HIF-1, immunohistochemistry

\section{Introduction}

Glaucoma is an eye disorder resulting in the loss of optic nerve and retinal ganglion cells that leads to characteristic optic neuropathy blindness. The most important risk factor is intraocular increased pressure. There are many different subtypes of glaucoma (including 'open angle' and 'closed angle' glaucoma), but they can all be considered as types of optic neuropathy [1]. Hypoxia in the optic nerve and retina is thought to develop secondary pressure in glaucomatous eyes, and is thought to be associated with pathogenic mechanisms underlying optic nerve and retinal ganglion cells degeneration in glaucoma. Improper blood circulation in the retina and optic nerve has been suggested as leading to retinal ganglion cell degeneration and death in glaucoma [2].

Correspondence address: J. Reszec, Department of Medical Pathomorphology, Medical University of Bialystok, Waszyngtona Str. 13, 15-269 Bialystok, Poland; e-mail: joasia@umb.edu.pl
Hypoxia-inducible factor 1 (HIF-1) is an oxygenregulated transcription factor that regulates oxygen homeostasis. Under hypoxic conditions, HIF-1 regulates the activation of various genes, including those encoding erythropoietin, glucose transporters, vascular endothelial growth factor and other genes, which increase oxygen supply or increase metabolic adaptation to the hypoxic condition. HIF is a heterodimeric transcription factor composed of one of the three oxygen-sensitive HIF-alpha subunits. In normoxic conditions, HIF-alpha is bound to von Hippel-Lindau protein and is damaged by ubiquitination and proteosomal degradation. Specifically, recent results have shown that the HIF pathway can be used as a therapeutic target, although there is still a long way to go from bench to clinic [3-5].

HIF-1 expression in optic nerve axons and in retinal ganglion cells has been presented in various studies; however, results are still equivocal. The aim of our study was to evaluate HIF-1 expression in retinal ganglion cells and optic nerve axons in glaucomatous eyeballs. 
Table 1. HIF-1 expression in optic nerve and retinal ganglion cells in glaucoma

\begin{tabular}{|l|c|c|c|c|}
\hline $\begin{array}{l}\text { HIF-1 } \\
\text { expression }\end{array}$ & $\begin{array}{c}- \\
\text { Number (\%) }\end{array}$ & $\begin{array}{c}+ \\
\text { Number (\%) }\end{array}$ & $\begin{array}{c}+++ \\
\text { Number (\%) }\end{array}$ & Total number (\%) \\
\hline Glaucomatous optic nerve & $18(42.9)$ & $19(45.2)$ & $5(11.9)$ & $42(100)$ \\
\hline Retinal ganglion cells & $20(47.6)$ & $20(47.6)$ & $2(4.8)$ & $42(100)$ \\
\hline
\end{tabular}

\section{Material and methods}

A total of 42 eyeballs were examined and enucleated in the Department of Ophthalmology of the Medical University of Bialystok over the period 2000-2010. All eyeballs were removed from patients with absolute glaucoma, who suffered from severe ophthalmalgia. The eyes were included in the study in accordance with the Declaration of Helsinki for research involving human tissue. Two eyeballs were taken as a control to the glaucoma cases; both were enucleated on the same day as the trauma.

After enucleation, the eyeballs were fixed in a $10 \%$ buffered formalin solution, embedded in paraffin at $56^{\circ} \mathrm{C}$, then cut into $5 \mu \mathrm{m}$ slices and stained with hematoxylin and eosin $(\mathrm{H}+\mathrm{E})$. Following deparaffinization, endogenous peroxidase activity was quenched by $3 \%$ hydrogen peroxide in methanol for 60 minutes. Nonspecific staining was prevented by pre-incubation with normal blocking serum. The sections were then incubated with mouse monoclonal antibody for HIF-1 $\alpha$ (sc 53546, Santa Cruz Biotechnology, Santa Cruz, CA, USA) in 1:75 dilutions, overnight at $4^{\circ} \mathrm{C}$ and labeled with EnVision (DAKO) enzyme reagent and diaminobenzidine (DAB) chromogen for 5 minutes.

Sections incubated without primary antibody were prepared as the negative control. Renal clear cell carcinoma specimens, which strongly express HIF-1 were taken as positive control.

Two independent pathologists performed the immunohistochemical evaluations of HIF-1. The estimation of immunostaining was done under a light microscope in representative fields at a magnification of $20 \times$. Specimens were considered immunopositive when $10 \%$ or more of the axons and retinal ganglion cells were clearly labeled HIF-1. The obtained results were statistically analyzed using Spearman's and Pearson's tests.

\section{Results}

The age for the glaucoma group ranged between 22-87 years, with 32 men and ten women. Five patients from the glaucoma group were suffering from hypertension, and five patients from coronary disease. Both patients with eye trauma were otherwise healthy.

HIF-1 expression was observed not only in the nuclear region of retinal ganglion cells, but a strong granular cytoplasmic reaction was also observed both in retinal ganglion cells and in optic nerve axons. (Table 1).

HIF-1 expression was observed in 24 out of 42 cases $(57.1 \%)$ of optic nerve axons of glaucomatous eyeballs; 19 of them $(45.2 \%)$ presented weak but diffused reaction categorized as $(+)$; five of the positive cases $(11.9 \%)$ were categorized as $(++)$ because of very strong, diffused HIF-1 immunostaining (Figure 1).

In the retinal ganglion cells, HIF-1 expression was noticed mostly in the nuclear and perinuclear region as a granular staining (Figures 2, 3). 22 out of 42 cases $(52.3 \%)$ were positive for HIF-1, and two of them presented very strong granular expression categorized as $(++)$. In 20 out of 42 cases (47.6\%), we observed HIF-1 positive immunohistochemical reaction both in retinal ganglion cells and optic nerve axons.

Retinal ganglion cells and the optic nerve axons taken from the trauma injury eyeballs did not present HIF-1 expression (Figure 4).

\section{Discussion}

In glaucomatous optic nerve axons and retinal ganglion cells, increased apoptosis is often observed as a result of increased intraocular pressure, causing irreversible damage to the optic nerve axons and, in consequence, resulting in vision loss. The major role in this pathway is assigned to proapoptotic proteins such as Bak and Bax, as well as enzymes like caspase-3 [6]. However it might also be a consequence of the activation of other factors such as HIF-1, which might occur in glaucomatous eyes as a consequence of hypoxia.

Our present study showed that nearly half of the examined cases presented HIF-1 expression, both in retinal ganglion cells and optic nerve axons. Strong HIF-1 immunoexpression was observed both in the glaucomatous optic nerves and in the nuclear and perinuclear region of the retinal ganglion cells. No injured eyeball presented HIF-1 expression, either in the retinal ganglion cells or in the optic nerve axons. It is very difficult to find unequivocal results in recent studies. Similarly to our study, Tezel et al. also found an increase in immunostaining for HIF-1 alpha in the retina and optic nerve head of glaucomatous donor eyes compared to control eyes, concluding that tissue hypoxia is present in the retina and 


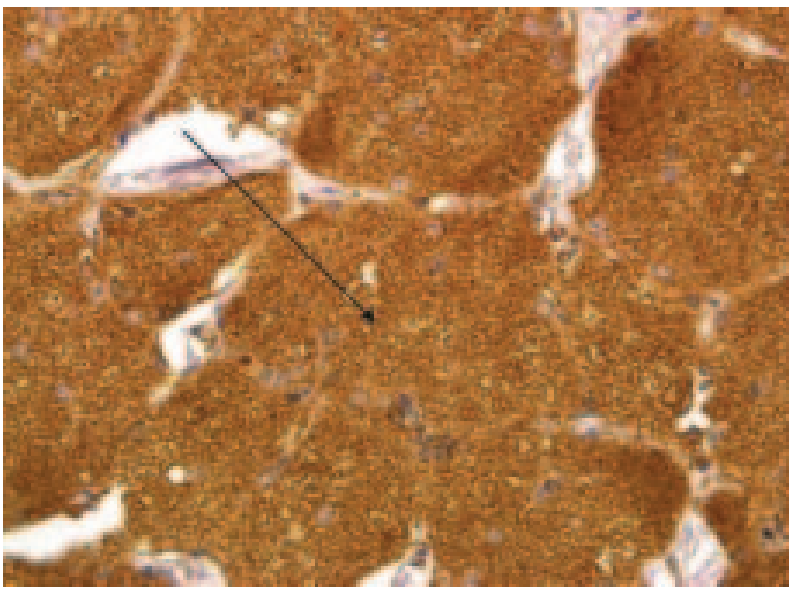

Figure 1. Optic nerve cross-section presenting strong HIF-1 expression observed in optic nerve axons. Magnification $\times 200$

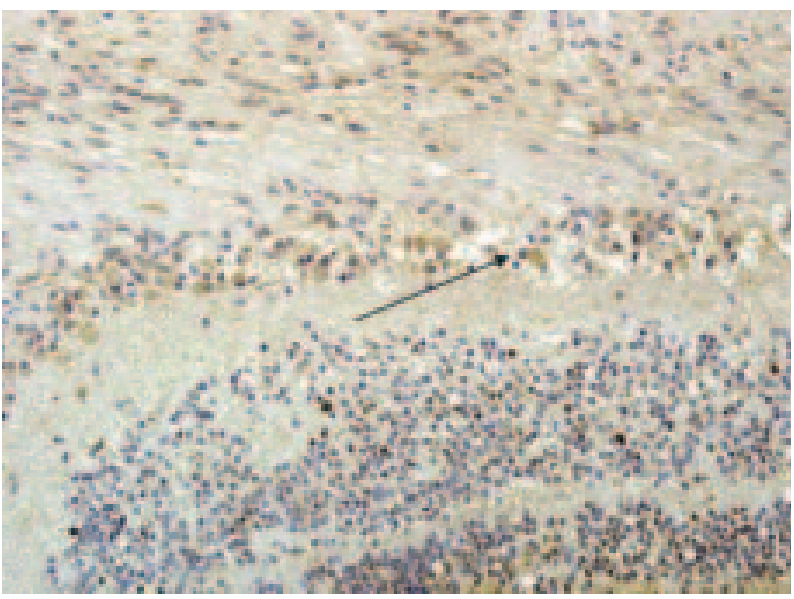

Figure 3. Strong HIF-1 expression observed in retinal ganglion cells compared to other retinal layers in a glaucomatous eye. Magnification $\times 100$

optic nerve head of glaucomatous eyes, and hypoxic signaling is a likely component of the pathogenic mechanisms of glaucomatous neurodegeneration [2]. Also, Mowat et al. observed strong HIF-1 expression in the neuronal cells through the inner retinal layers during hypoxia [7]. Gui et al. presented significantly decreased HIF-1 mRNA expression after erythropoietin treatment, suggesting its protective role to the injured retina by chronic intraocular hypertension [5].

Completely different results have been presented Savagian et al., who observed that neither control eyes nor specimens from the eyes with acute glaucoma had nuclear staining for HIF-1alpha in the retinas [8]. We find it very interesting that Zhu et al. [9] observed decreased HIF-1 expression in retinal ganglion cells after pilocarpine treatment of glaucomatous eyes, suggesting, similarly to Arjamaa et al. [3], that the HIF-1 alpha pathway as a 'master switch' could be

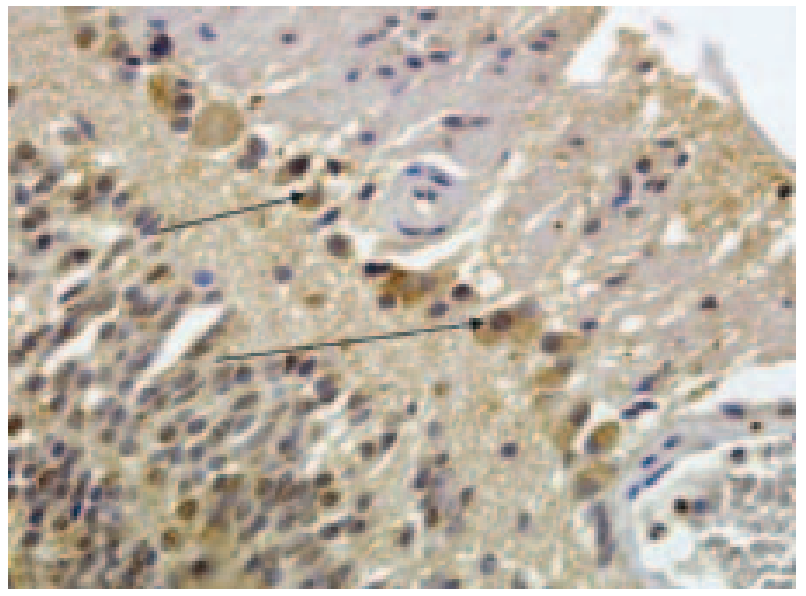

Figure 2. HIF-1 protein expression observed in retinal ganglion cells in a glaucomatous eye. Magnification $\times 400$

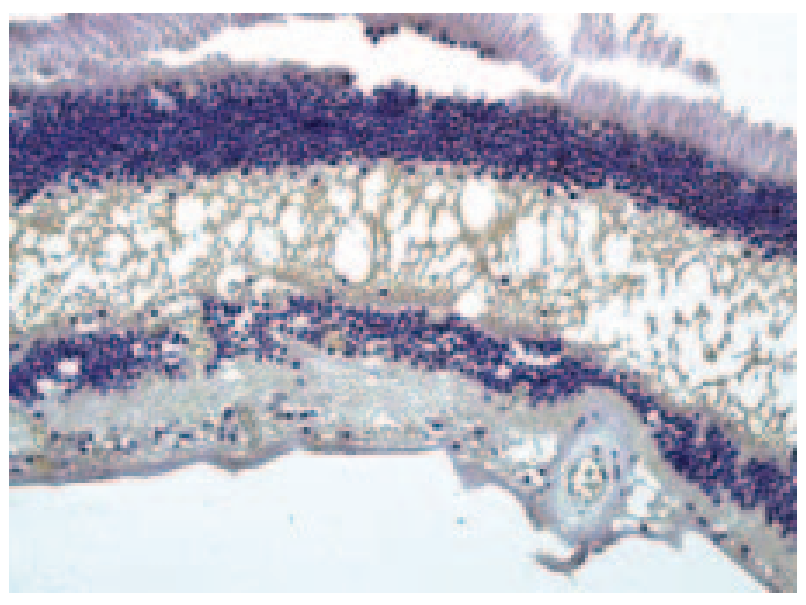

Figure 4. Eyeball enucleated on the day of injury. Retinal ganglion cells and other retinal layers presenting negative immunostaining for HIF-1. Magnification $\times 100$

used as a therapeutic target in the treatment of glaucoma. Whitlock et al. discovered that HIF-1 activates shock proteins, including Hsp27, protecting the rat retina from ischemic injury [10]. These findings were significant, because it was the first time HIF-1 activation has been associated with the protective effects of the retinal ganglion cells in glaucoma.

Our present results and other studies show that HIF-1 expression in hypoxic conditions in glaucoma might be a very crucial stage in retinal ganglion cells and optic nerve axons damage, and might be a successful target for the implementation of neuroprotective drugs.

\section{References}

1. Prasanna G, Krishnamoorthy R, Yorio T. Endothelin, astrocytes and glaucoma. Experimental Eye Research. 2011;93:170-177. 
2. Tezel G, Wax MB. Hypoxia-inducible factor 1alpha in the glaucomatous retina and optic nerve head. Arch Ophthalmol. 2004;122:1348-1356.

3. Arjamaa O, Nikinmaa M. Oxygen-dependent diseases in the retina: role of hypoxia-inducible factors. Exp Eye Res. 2006;83:473-483.

4. Ergorul C, Ray A, Huang W et al. Hypoxia inducible factor$1 \alpha(\mathrm{HIF}-1 \alpha)$ and some HIF-1 target genes are elevated in experimental glaucoma. J Mol Neurosci. 2010;42:183-191.

5. Gui DM, Yang Y, Li X, Gao DW. Effect of erythropoietin on the expression of HIF-1 and iNOS in retina in chronic ocular hypertension rats. Int J Ophthalmol. 2011;4:40-43.

6. Zalewska R, Zalewski B, Reszec J, Mariak Z, Zimnoch L, Proniewska-Skretek E. The expressions of Fas and caspase-3 in human glaucomatous optic nerve axons. Med Sci Monit. 2008;14:274-278.
7. Mowat FM, Luhmann UFO, Smith A et al. HIF-1alpha and HIF-2alpha Are Differentially Activated in Distinct Cell Populations in Retinal Ischaemia. PLoS One. 2010;14;5:11103.

8. Savagian CA, Dubielzig RR, Nork TM. Comparison of the distribution of glial fibrillary acidic protein, heat shock protein 60, and hypoxia-inducible factor-1alpha in retinas from glaucomatous and normal canine eyes. Am J Vet Res. 2008;69:265-272.

9. Zhu X, Zhou W, Cui Y et al. Pilocarpine protects cobalt chloride-induced apoptosis of RGC-5 cells: involvement of muscarinic receptors and HIF-1 alpha pathway. Cell Mol Neurobiol. 2010;30:427-435.

10. Whitlock NA, Agarwal N, Ma JX, Crosson CE. Hsp27 upregulation by HIF-1 signaling offers protection against retinal ischemia in rats. Invest Ophthalmol Vis Sci. 2005; 46:1092-1098.

Submitted: 13 December, 2011

Accepted after reviews: 27 April, 2012 